\title{
Codein: Neue Anwendungsbeschränkungen bei Kindern und Jugendlichen
}

Julia C. Stingl, Jens Rotthauwe

Das Schmerzmittel und Antitussivum Codein kann vor allem bei Kindern lebensgefährliche Nebenwirkungen verursachen. Daher sind codeinhaltige Medikamente laut einer aktuellen Mitteilung des Bundesinstitut für Arzneimittel und Medizinprodukte für Kinder unter 12 Jahren kontraindiziert. Auch bei Patienten im Alter von 12-18 Jahren wird der Einsatz des Medikaments wegen erhöhter Anfälligkeit für Atemstörungen nicht mehr empfohlen. Vorsicht ist aber auch bei Erwachsenen geboten, die den Wirkstoff sehr schnell zum Morphin verstoffwechseln [1, 2].

\section{Hintergrund}

Todesfälle durch Codein I Es ist ein trauriger Anlass, der zu den aktuellen Warnungen und Markteinschränkungen von Codein führte: In mehreren Fällen, darunter einer in Deutschland, starben Kinder an einem plötzlichen Atemstillstand, nachdem sie codeinhaltige Tropfen gegen ihren Husten bekommen hatten. Ein Zusammenhang fiel zunächst bei einem Neugeboren auf, dessen Mutter nach einem Kaiserschnitt Codein gegen die Schmerzen erhielt. Im Nachhinein stellte man fest, dass die Mutter wegen eines pharmakogenetischen Polymorphismus des Enzyms CYP2D6 ultraschnell Codein zu Morphin umgewandelt hatte. Dieses nahm das Kinder über die Muttermilch auf [3].

Atemdepression oft schwer zu erkennen I Bei Kindern und insbesondere bei Neugeborenen als "vulnerable Patientengruppe" ist die Bildungsund Ausscheidungskapazität der Nieren für glucuronidiertes Morphin noch vermindert. Dadurch wird es schlechter eliminiert und es treten besonders häufig Nebenwirkungen auf [4].

Zudem verursacht Morphin eine zentrale Atemdepression, die unter Umständen (z. B. wenn sie nachts auftritt und bei kleinen Kindern) schwer zu erkennen ist.

Diese erkennbaren Arzneimittel-Risiken und die pharmakogenetische Ursache führten dazu, dass ein vom BfArM angestoßenes europäisches Risikobewertungsverfahren eingeleitet wurde. Nun gab das BfArM die Anwendungsbeschränkungen für Codein in ganz Europa bekannt [1, 2].

\section{Pharmakogenetischer Polymorphismus bei CYP2D6}

Die Rolle von CYP2D6 | Verantwortlich für die Verstoffwechselung von Codein - sowie ver- schiedener anderer Arzneimittel und Fremdstoffe - ist ein körpereigenes Leberenzym der Cytochrom-P450-Familie, das CYP2D6. Dieses Enzym baut Medikamente und Fremdstoffe mehr oder weniger schnell in unserem Körper ab [5]. Die Geschwindigkeit ist genetisch bedingt - es liegt ein pharmakogenetischer Polymorphismus zugrunde.

Unterschiedlich hohe Enzymaktivität | Wie aktiv CYP2D6 ist, ist von Mensch zu Mensch unterschiedlich. Bei etwa 7\% unserer Bevölkerung fehlt das Enzym sogar ganz. Bei diesen Personen ist nach Codein-Einnahme kein Morphin im Blut nachweisbar und es tritt auch keine analgetische Wirkung ein [6]. Im Gegensatz dazu verstoffwechseln etwa 3\% der Bevölkerung ultraschnell. Dies wurde vor Jahren in Schweden bei der Familie einer Krankenschwester beobachtet, die selbst in der klinischen Forschung tätig war. Sie beobachtete, dass sie sehr hohe Dosierungen mancher Arzneimittel gut vertrug und offenbar sehr schnell abbaute. Als Ursache identifizierte man eine Genduplikation - in ihrem Fall lag das Gen sogar in 13-facher Ausführung vor [7]. Dies führt dazu, dass CYP2D6 sehr stark exprimiert ist und mit hoher Aktivität verschiedene Arzneimittel sehr schnell abbauen kann ("Ultraschnellmetabolismus“).

Betroffene Patienten fallen meist dadurch auf, dass sie entweder höhere Dosierungen für eine erwünschte Wirkung benötigen, oder dass sie mehr Nebenwirkungen haben - z. B. bei Codein.

Wie viel Prozent der Bevölkerung zu den ultraschnellen Metabolisierern gehören, ist regional unterschiedlich. In den asiatischen Ländern ist der Polymorphismus nicht so ausgeprägt und es gibt weder die kompletten "langsam Metabolisierer" noch die "ultraschnell Metabolisierer" [8]. Demgegenüber wurde in arabischen Ländern oder in Äthiopien gezeigt, dass etwa 1/3 der Bevölkerung 
"Ultraschnell-Metabolisierer" sind und eine Genduplikation aufweisen $[9,10]$.

Dosierung anpassen | Es stellt sich die Frage, welche Rolle der Polymorphismus für Arzneimittel, die über CYP2D6 verstoffwechselt werden, sonst noch spielt. Die großen Unterschiede in der Pharmakokinetik haben schon in der Vergangenheit dazu geführt, dass durch entsprechende Dosierungsanpassungen versucht wurde, eine ähnliche Exposition an Arzneimitteln zu erreichen [11]. Es liegt nahe, die Dosierungen so zu adaptieren, wie es beispielsweise bei älteren Patienten oder bei Niereninsuffizienz gemacht wird. Bei einem langsamen Metabolisierer sollte man die Dosis entsprechend reduzieren und bei ultraschnellen Metabolisierern gegebenenfalls erhöhen.

Alternative Behandlung I Genetische Polymorphismen in der täglichen Praxis zu beachten, ist in den Fällen leichter, in denen das Enzym CYP2D6 ein Arzneimittel einfach nur inaktiviert. Wenn ein Patient zu langsam inaktiviert, kann man die Dosis entsprechend anpassen. Das Ganze wird aber komplizierter, wenn ein Arzneimittel - wie das Codein - erst aktiviert wird und der Metabolit Atemstörungen bis hin zum Atemstillstand verursachen kann. In diesem Fall bringt auch eine vorsichtige Dosierung wenig, denn auch dann kann Codein schnell und vollständig zu Morphin umgewandelt werden.

Somit sollten „Ultraschnell-Metabolisierer“ alternative Behandlungsoptionen für Husten oder Schmerzen in Betracht ziehen $[12,13]$.

\section{Forschungsaktivitäten des BfArM}

Aktivität von CYP2D6 im Gehirn | Derzeit führt das BfArM gemeinsam mit dem Karolinska Institutet Stockholm und der Universität Toronto ein aktuelles europäisches Forschungsprojekt durch (ERANET Neuron „BrainCYP“). Dieses zielt darauf ab, die Aktivität von CYP2D6, das nicht nur in der Leber, sondern auch in neuronalen Zellen vorkommt, im lokal aktiven Stoffwechsel des Gehirns zu untersuchen. Es geht um die Frage, inwieweit die starke Expression von CYP2D6 und der lokale Metabolismus im Gehirn für zentrale Wirkungen und Nebenwirkungen von Arzneimitteln wie Codein, Antidepressiva, Antipsychotika oder bestimmten Antihistaminika von Bedeutung sind. Außerdem wird untersucht, in welcher Weise der Polymorphismus den lokalen Gehirnstoffwechsel beeinflusst.

Höhere Wahrscheinlichkeit für Suizid I Möglicherweise kann CYP2D6 zentrale Nebenwirkungen oder auch die Biotransformation von körpereigenen Substanzen beeinflussen. So gibt es eine Reihe von Studien, die bei depressiven Patienten zeigten, dass "Ultraschnell-Metabolisierer" unter Umständen eine höhere Neigung zu suizidalen Gedanken oder Handlungen haben. Dies wurde zuerst in einer Studie aus Schweden an Suizidopfern untersucht $[14,15]$. Unter den Opfern lag im Vergleich zum Kontrollkollektiv eine deutlich höhere Häufigkeit der Genduplikation von CYP2D6 vor. Es wurde vermutet, dass die Verstorbenen das Antidepressivum zu schnell verstoffwechselten, so dass es nicht richtig wirken konnte.

CYP2D6 und Neurotransmitter | Davon unabhängige Studien zeigen, dass auch bei Patienten mit Essstörungen sowie bei depressiven Patienten eine möglicherweise generell höhere Neigung zu suizidalen Gedanken oder Handlungen mit diesem genetischen Polymorphismus assoziiert war [16-19].

Möglicherweise ist CYP2D6 nicht nur für Metabolisierung von Arzneistoffen wichtig, sondern auch für die Biotransformation von körpereigenen Substanzen, z. B. von Neurotransmittern.

Möglicherweise beeinflusst das Enzym bestimmte Schritte im Serotonin- oder auch Dopaminstoffwechsel, was zu affektiven Symptomen oder impulsgesteuerten Handlungen führen könnte [20].

Ist CYP2D6 induzierbar? I Im Forschungsprojekt des BfArM wird mittels neuronaler Bildgebung (fMRT) der genetische Polymorphismus von CYP2D6 im Gehirn genauer untersucht. Darüber hinaus finden Untersuchungen zur Rolle des Hirnmetabolismus für Nebenwirkung und Wirkung von Arzneimitteln statt. Die an diesem Projekt beteiligte kanadische Gruppe konnte zeigen, dass CYP2D6 im Gehirn möglicherweise induzierbar ist - die Aktivität also nochmal erhöht werden kann [21]. Durch Induktion würde ein Patient zum ultraschnellen Metabolisierer werden, auch wenn er ansonsten Codein normal verstoffwechselt.

Einfluss von Nikotin und Alkohol | Bisher geht man davon aus, dass CYP2D6 nicht zu den induzierbaren Enzymen zählt, da es von den üblichen Enzyminduktoren wie Barbituraten oder Carbamazepin nicht betroffen ist. Bei Rauchern und auch bei chronischen Suchtpatienten mit Alkoholabhängigkeit ist die Aktivität von CYP2D6 im Gehirn jedoch erhöht [22]. Grund hierfür könnten epigenetische Mechanismen sein sowie Unterschiede im Abbau des Enzyms oder in der Abbaugeschwindigkeit, die dazu führen, dass netto die Enzymaktivität erhöht ist [21]. An Ratten wurde jetzt gezeigt, dass der Codeinstoffwechsel im Gehirn beschleunigt wird, wenn Nikotin über eine längere Zeit infundiert wird [23]. Interessanterweise war in den bisherigen Untersuchungen die Veränderung durch Nikotin nur für das im Gehirn vorkommende CYP2D6- 
Enzym nachweisbar - das Leberenzym wurde nicht vermehrt gefunden [23].

\section{Individualisierte Dosisempfehlungen}

Guidelines für Dosierungsanpassungen | Dosisempfehlungen, die auf pharmakogenetischen Parametern basieren, werden bereits angewandt und weiterentwickelt [11, 24-26]. Von den USA aus wird ein internationales Consortium koordiniert, das sich mit der Erstellung von solchen Therapieempfehlungen beschäftigt („Clinical Pharmacogenomics Implementation Consortium“) $[26,27]$. Hier werden unter anderem für das Enzym CYP2D6 Guidelines herausgegeben, die Dosierungsanpassungen vorschlagen, die auf einer Auswertung sämtlicher vorhandener Daten zur Pharmakokinetik basieren. Dazu gehören beispielsweise trizyklische Antidepressiva oder auch selektive Serotonin-Wiederaufnahmehemmer [28, 29].

Anpassung von Packungsbeilagen | Pharmakogenetische Informationen werden somit auch für die tägliche Praxis immer wichtiger. Sie finden sich mittlerweile in vielen Fachinformation und Packungsbeilagen verschiedener Arzneimittel bald auch bei Codein. Seitens der amerikanischen Zulassungsbehörde (FDA) sind schon über 120 Arzneimittel um Pharmakogenetik-Informationen ergänzt worden [30]. In den medizinischen Fachinformationen auf europäischer Ebene sind es mittlerweile schon über 70 Arzneimittel [31].

Blick in die Zukunft | Wir erwarten, dass in Zukunft durch genetische oder molekulare Marker eine noch genauere und besser stratifizierte Einschätzung des Nutzen-Risikoverhältnisses bei Arzneimitteln für verschiedene Patientengruppen im Sinne einer „individualisierte Medizin“ möglich wird.

\section{Konsequenz für Klinik und Praxis}

- Codein ist für die Anwendung bei Kindern unter 12 Jahren kontraindiziert.

- Zur Behandlung von Husten bei Kindern und Jugendlichen zwischen 12 und 18 Jahren mit ausgeprägten Atemwegsbeeinträchtigungen wird der Einsatz des Medikaments nicht empfohlen [1].

- Codein darf während der Stillzeit nicht angewendet werden.

- Codein ist bei Patienten kontraindiziert, von denen bekannt ist, dass sie ultraschnelle CYP2D6-Metabolisierer sind [1]

- Pharmakogenetische Informationen aus Packungsbeilagen und Fachinformationen sollen Sie in der täglichen Praxis beachten.

- Unerwünschte Arzneimittelwirkungen (UAW) sollen dem BfArM gemeldet werden.

\section{Literatur}

1 Bundesinstitut für Arzneimittel und Medizinprodukte (BfArM). Pressemitteilung zu Codein (24.04.2015). Im Internet: http://www.bfarm.de/ SharedDocs/Pressemitteilungen/DE/mitteil2015; Stand: 27.05.2015

2 Bundesinstitut für Arzneimittel und Medizinprodukte (BfArM). Codein: Anwendungsbeschränkungen zur Behandlung von Kindern und Jugendlichen mit Husten; Empfehlung des europäischen Ausschusses für Risikobewertung (PRAC) bestätigt (24.04.2015). Im Internet: http://www.bfarm.de/SharedDocs/ Risikoinformationen/Pharmakovigilanz/DE/ RV_STP/a-f/codein3.html; Stand: 27.05.2015)

3 Koren G, Cairns J, Chitayat D et al. Pharmacogenetics of morphine poisoning in a breastfed neonate of a codeine-prescribed mother. Lancet 2006; 368: 704

4 Knibbe CA, Krekels EH, van den Anker JN et al. Morphine glucuronidation in preterm neonates, infants and children younger than 3 years. Clin Pharmacokinet 2009; 48: 371-385

5 Zanger UM, Raimundo S, Eichelbaum M. Cytochrome P450 2D6: overview and update on pharmacology, genetics, biochemistry. Naunyn Schmiedebergs Arch Pharmacol 2004; 369: 23-37

6 Kirchheiner J, Schmidt H, Tzvetkov M et al. Pharmacokinetics of codeine and its metabolite morphine in ultra-rapid metabolizers due to CYP2D6 duplication. Pharmacogenomics J 2007; 7: 257-265

7 Johansson I, Lundqvist E, Bertilsson L et al. Inherited amplification of an active gene in the cytochrome P450 CYP2D locus as a cause of ultrarapid metabolism of debrisoquine. Proc Natl Acad Sci USA 1993; 90: 11825-11829

8 LLerena A, Naranjo ME, Rodrigues-Soares F et al. Interethnic variability of CYP2D6 alleles and of predicted and measured metabolic phenotypes across world populations. Expert Opin Drug Metab Toxicol 2014; 10: 1569-1583

9 Aklillu E, Persson I, Bertilsson L et al. Frequent distribution of ultrarapid metabolizers of debrisoquine in an ethiopian population carrying duplicated and multiduplicated functional CYP2D6 alleles. J Pharmacol Exp Ther 1996; 278: 441-446

10 McLellan RA, Oscarson M, Seidegard J et al. Frequent occurrence of CYP2D6 gene duplication in Saudi Arabians. Pharmacogenetics 1997; 7 : 187-191

11 Kirchheiner J, Fuhr U, Brockmoller J. Pharmacogenetics-based therapeutic recommendations ready for clinical practice? Nat Rev Drug Discov 2005; 4: 639-647

12 Crews KR, Gaedigk A, Dunnenberger HM et al. Clinical Pharmacogenetics Implementation Consortium (CPIC) guidelines for codeine therapy in the context of cytochrome P450 2D6 (CYP2D6) genotype. Clin Pharmacol Ther 2012; 91: 321-326

13 Crews KR, Gaedigk A, Dunnenberger HM et al. Clinical Pharmacogenetics Implementation Consortium guidelines for cytochrome P450 2D6 genotype and codeine therapy: 2014 update. Clin Pharmacol Ther 2014; 95: 376-382

14 Zackrisson AL, Holmgren P, Gladh AB et al. Fatal intoxication cases: cytochrome P450 2D6 and 2C19 genotype distributions. Eur J Clin Pharmacol 2004; 60: 547-552

15 Zackrisson AL, Lindblom B, Ahlner ]. High frequency of occurrence of CYP2D6 gene duplication / multiduplication indicating ultrarapid metabolism among suicide cases. Clin Pharmacol Ther 2010; 88: 354-359

Vollständiges Literaturverzeichnis unter http://dx.doi.org/10.1055/s-0041-102948

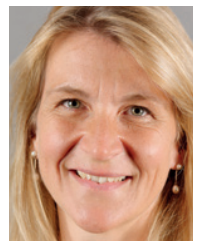

Prof. Dr. med. Julia C. Stingl ist Vizepräsidentin und Leiterin der Abteilung Forschung am Bundesinstitut für Arzneimittel und Medizinprodukte.

julia.stingl@bfarm.de

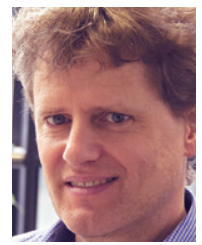

Jens Rotthauwe

ist wissenschaftlicher Mitarbeiter der Abteilung Pharmakovigilanz im Bundesinstitut für Arzneimittel und Medizinprodukte.

Interessenkonflikt

Die Autorin gibt an, dass kein Interessenkonflikt besteht.

DOI 10.1055/s-0041-102948

Dtsch Med Wochenschr 2015; 140: 1093-1095 C Georg Thieme Verlag KG . Stuttgart · New York · ISSN 0012-0472 
16 Druid H, Holmgren P, Carlsson B et al. Cytochrome P450 2D6 (CYP2D6) genotyping on postmortem blood as a supplementary tool for interpretation of forensic toxicological results. Forensic Sci Int 1999; 99: 25-34

17 Holmgren P, Carlsson B, Zackrisson AL et al. Enantioselective analysis of citalopram and its metabolites in postmortem blood and genotyping for CYD2D6 and CYP2C19. J Anal Toxicol 2004; 28: 94-104

18 Stingl JC, Viviani R. CYP2D6 in the brain: impact on suicidality. Clin Pharmacol Ther 2011; 89: 352-353

19 Penas-LLedó EM, Dorado P, Aguera Z et al. CYP2D6 polymorphism in patients with eating disorders. Pharmacogenomics J 2012; 12: 173-175

20 Bertilsson L. CYP2D6, serotonin, and suicide - a relationship? Clin Pharmacol Ther 2011; 88: 304-305

21 Mann A, Miksys S, Lee A et al. Induction of the drug metabolizing enzyme CYP2D in monkey brain by chronic nicotine treatment. Neuropharmacology 2008; 55: 1147-1155

22 Miller RT, Miksys S, Hoffmann E et al. Ethanol self-administration and nicotine treatment increase brain levels of CYP2D in African green monkeys. Br J Pharmacol 2014; 171: 3077-3088

23 McMillan DM, Tyndale RF. Nicotine Increases Codeine Analgesia Through the Induction of Brain CYP2D and Central Activation of Codeine to Morphine. Neuropsychopharmacology 2015; 40: 1804-1812

24 Kirchheiner J, Brosen K, Dahl ML et al. CYP2D6 and CYP2C19 genotype-based dose recommendations for antidepressants: a first step towards subpopulation-specific dosages. Acta Psychiatr Scand 2001; 104: 173-192
25 Kirchheiner J, Nickchen K, Bauer M et al. Pharmacogenetics of antidepressants and antipsychotics: the contribution of allelic variations to the phenotype of drug response. Mol Psychiatry 2004; 9: 442-473

26 Swen J], Nijenhuis M, de Boer A et al. Pharmacogenetics: from bench to byte - an update of guidelines. Clin Pharmacol Ther 2011; 89: 662-673

27 Relling MV, Klein TE. CPIC: Clinical Pharmacogenetics Implementation Consortium of the Pharmacogenomics Research Network. Clin Pharmacol Ther 2011; 89: 464-467

28 Biernacka JM, Sangkuhl K, Jenkins G et al. The International SSRI Pharmacogenomics Consortium (ISPC): a genome-wide association study of antidepressant treatment response. Translational psychiatry 2015; 5: e553

29 Hicks JK, Swen JJ, Thorn CF et al. Clinical Pharmacogenetics Implementation Consortium guideline for CYP2D6 and CYP2C19 genotypes and dosing of tricyclic antidepressants. Clin Pharmacol Ther 2013; 93: 402-408

30 Frueh FW, Amur S, Mummaneni P et al. Pharmacogenomic biomarker information in drug labels approved by the United States food and drug administration: prevalence of related drug use. Pharmacotherapy 2008; 28: 992-998

31 Ehmann F, Caneva L, Prasad K et al. Pharmacogenomic information in drug labels: European Medicines Agency perspective. Pharmacogenomics | 2015; 15: 201-210 